\title{
Pressurized Liquid Extraction of Hemp Residue and Purification of the Extract with Liquid-Liquid Extraction
}

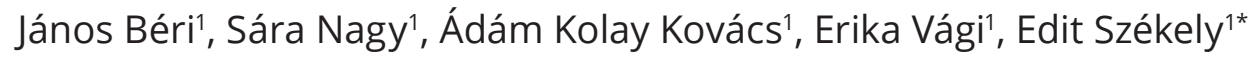 \\ 1 Department of Chemical and Environmental Process Engineering, Faculty of Chemical Technology and Biotechnology, Budapest \\ University of Technology and Economics, H-1111 Budapest, Müegyetem rkp. 3., Hungary \\ * Corresponding author, e-mail: edit.szekely@edu.bme.hu
}

Received: 30 April 2021, Accepted: 14 July 2021, Published online: 19 November 2021

\begin{abstract}
The first semi-continuous Pressurized Liquid Extraction (PLE) of hemp threshing residue with ethanol was carried out according to a $3^{2}$ full factorial experimental design with pressure and temperature as independent variables at 8-10-12 MPa and 323-333-343 K, respectively. The total- and cannabidiol (CBD) yield curves were fitted to the modified two-parameter Brunner equation. Best results, concerning CBD, can be achieved at $12 \mathrm{MPa}$ and $343 \mathrm{~K}$. Solvent mass-consumption and operation time were considerably decreased compared to a previous supercritical fluid extraction study on the same material. Furthermore, the concentration profiles were evaluated to study the mass transfer. The winterized dry extracts were further studied in a methanol-hexane-water ternary system concerning CBD distribution ratio, showing high methanol dependency.
\end{abstract}

Keywords

hemp threshing residue, solid-liquid extraction, PLE, CBD, distribution coefficient, ternary mixture

\section{Introduction}

Cannabis sativa L. is a well-known plant for either as textile fiber or its recreational use. The origin of it is most likely in Central North-East Asia [1]. As fiber material, it is proved to be at least 5000 years old. Knowledge on its psychotropic effect dates back 2200 years in one of the oldest pharmacopeias, the Pen-ts'ao Ching, states the excessive use of the "fruits of hemp" causes hallucinations, precisely: "seeing devils" [2].

Due to human impact, two main phenotypes had developed from the species. One is marijuana a highly psychoactive and the other is industrial hemp a fiber-type variety. Both phenotypes are bioresource of phytocannabinoids a diverse group of isoprenylated polyketides. [3] There are more than 90 cannabinoids that can be present in the plant. Practical classification differentiates two groups: psychotropic cannabinoids (PC) and non-psychotropic cannabinoids (NPC). $\Delta^{9}$-tetrahydrocannabinol ( $\left.\Delta^{9}-\mathrm{THC}\right)$, its acid (THCA), and its oxidized form cannabinol (CBN) are the most charted PC compounds. Cannabidiol (CBD), its acid (CBDA), are the most influential NPCs. The composition of the cannabinoid profile of each plant phenotypes can vary to a great extent on weather and soil conditions [4-6]. Psychoactive-type cultivation focuses on PC enrichment in the plant. Industrial hemp - classification criteria: $\Delta^{9}$-THC content is lower than $0.3 \mathrm{w} / \mathrm{w} \%$; EU legislation [7] is mainly considered a fiber source $[4,6]$. It represents a high-quality fiber that is adequate for several industries such as the automotive industry, bio-building, paper manufacturing, and textile industry $[6,8]$.

In the fiber processing industry the inflorescences, leaves, and thin stems are called the threshing residue which is usually discarded or used for animal bedding - however, there are examples where the seeds are also considered as a waste [9]. These remaining waste materials might represent an opportunity. A study estimated that during seed harvest and seed cleaning $2 \mathrm{t} / \mathrm{ha}$ residue can be collected [5]. In another study, at a fiber production facility, lost hemp dust was evaluated to be $18-33 \mathrm{w} / \mathrm{w} \%$ of the raw material [10]. These different residues contain several valuable components like sugars, alkaloids, quinones [10], and NPCs some of which are specific to the genus Cannabis [3].

The aforementioned cannabidiol (CBD) has become the subject of considerable interest in recent years. Several studies proved its therapeutic effect for the treatment of the central nervous system, peripheral disorder, pain, and epilepsy without any psychotropic effect [11-14]. 
As fiber type hemp varieties are convenient sources of CBD, several studies were conducted to obtain it from the various parts of the harvested plant. Besides, antioxidants, aromas, essential oils, fatty acids etc. were also found in significant quantities [13-24]. However, in the practical application, the separation of solid fractions (e.g., inflorescences from leaves) is highly time-consuming and also unfavorable for the low-cost agricultural industry. In response, some recent studies focus on combined threshing residue extraction [4, 25-28], or stage-wise extraction of waste materials with respect to different fiber production units [8]. These separation processes are all based on solid-liquid or fluid extractions and mainly with batch operation.

Commonly used solvents for hemp extractions were methanol, ethanol, hexane, acetone, and supercritical carbon dioxide $\left(\mathrm{scCO}_{2}\right)$. In some cases, water or ethanol is added as a co-solvent. Solubility data is scarce since the composition and concentration profile of the same plant type varies yearly. Therefore the solubility data in these studies might contradict extraction data due to the matrix effect.

Several techniques are available for enhanced separation such as accelerated solvent extraction [29], microwaveor ultrasound-assisted extraction [15, 30], and Pressurized Liquid Extraction (PLE) for hemp extraction [31, 32]. From these techniques, the most promising for continuous or semi-continuous operation is the PLE.

Serna-Ioaiza et al. [32] carried out a three-step upscaling of hemp threshing residue extraction. A two-cycle batch PLE study was conducted at pressures (P) of 5-10-15 MPa, and temperatures $(\mathrm{T})$ at $323-348 \mathrm{~K}$ with a liquid-to-solid mass ratio of 3.3-5. Two-cycle extraction was rejected due to the low amount recovered in the second step. A twoway ANOVA of the extracts showed that the pressure did not influence either the CBD or $\Delta^{9}$-THC yields. Optimal extraction time in the PLE section was estimated between 10-100 min.

In another example, Kitrytė et al. [31] biorefined hemp threshing residue with consecutive supercritical carbon dioxide extraction, acetone PLE, water-ethanol PLE, and enzyme-assisted extraction. The best operational conditions of each stage were determined, and a thorough analysis of the products was reported for each stage. The optimal parameters of both PLE experiments were found to be 10.3 $\mathrm{MPa}$ and $373 \mathrm{~K}$ for $45 \mathrm{~min}$ while liquid to solid ratio was not specified.

In this study, the first semi-continuous PLE was carried out on hemp threshing residue with absolute ethanol. Two different total extraction times were examined.
The mass-transfer kinetics was further studied by evaluation of the concentration profiles. After evaluation of the PLE experimental design, the extracts were combined into a bulk, winterized, mixed then separated into five uniform parts. In five different hexane-methanol-water system the extracts were separately redissolved. The distribution ratio of $\mathrm{CBD}$ in theses systems was analyzed to further investigate the possible purification options of CBD from the crude extract.

\section{Materials and methods \\ 2.1 Materials}

For the measurements, acetonitrile (CAS: 75-05-8, purity: $>99.0 \%$ ), absolute ethanol (CAS: 64-15-5, purity: $\geq 99.5 \%$ ), methanol (CAS: 67-56-1; purity: $\geq 99.5 \%$ ), and hexane (CAS: 110-54-3, purity: $\geq 95.0 \%$ ) were purchased from Merck Millipore Ltd. (Budapest, Hungary). The ammonium-acetate (CAS: 631-61-8, purity: $\geq 97 \%$ ) and acetic-acid (CAS: 64-19-7, purity: $\geq 96.0 \%$ ) were purchased from Molar Chemicals Ltd. (Budapest, Hungary). Water was locally distilled with an electric conductivity of $0.1 \mathrm{mS} / \mathrm{cm}$. For filtration $0.02 \mu \mathrm{m}$ Whatman ${ }^{\mathrm{TM}}$ Anotop $^{\mathrm{TM}}$ inorganic (alumina based) membrane filter (syringe filter) was purchased from Merck. For winterization a glass filter was used with 10-16 $\mu \mathrm{m}$ porosity.

The industrial hemp phenotype was previously the subject of another study of our research group (marked as Sample 5) [25]. It is a fiber-type variaty harvested in 2013 with a moisture content of $11.0 \pm 0.1(\mathrm{w} / \mathrm{w} \%)$. The particle size is $0.50 \pm 0.01 \mathrm{~mm}$ with a uniformity factor of $2.05 \pm 0.06$. Total CBD content was evaluated to be $782 \pm 32 \mathrm{mg} / 100 \mathrm{~g}$ of dry material by Soxhlet extraction with acetonitrile.

\subsection{Methods}

\subsubsection{PLE experiments}

The Pressurized Liquid Extraction setup can be seen in Fig. 1. A $1 / 2$ stainless steel tube is used as an extractor. LKB Bromma 2150 HPLC pump is employed to provide the constant volumetric ethanol flow of $1.5 \mathrm{~mL} / \mathrm{min}$ a $3 \mathrm{~m}$ long 1/16' stainless steel capillary is connecting the extractor and the liquid pump.

After filling the extractor with approximately $7 \mathrm{~g}$ of threshing residue, the tube was closed with cotton balls on both ends then connected to the $1 / 16^{\prime}$ capillary and preheated for $5 \mathrm{~min}$ in the water bath. The maximal flow rate $(5.00 \mathrm{~mL} / \mathrm{min})$ was used to fill the extractor rapidly. After the first few drops, the regulating valve was closed until pressure build-up. Then, at the set flow rate, the pressure 


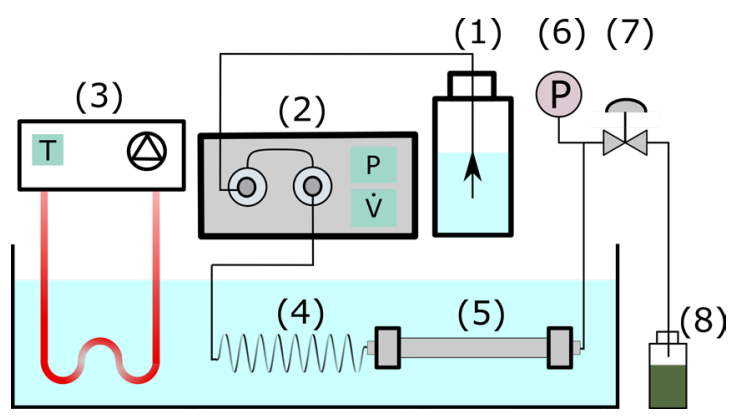

Fig. 1 Schematic representation of PLE experiment apparatus; (1) ethanol solvent, (2) HPLC pump, (3) heating element, (4) solvent pre-heating section, (5) $1 / 2$ ' extractor tube, (6) pressure gauge,

(7) hand-regulated valve, (8) sample vial

regulation was carried out manually and the isothermal conditions were kept by heated water bath. The preheating of the solvent was carried out in the same bath by submerging $2 \mathrm{~m}$ of the $1 / 16$ ' capillary.

The total sampling time for the runs were 5 and 20 minute with 1 and 5 minute sampling intervals, respectively. The weight of each sample were measured before and after evaporation using a BiotageTM TurboVap LV stripper at $318 \mathrm{~K}$ with compressed air until completely dry $\left(\dot{V}_{s t r}=3.5 \mathrm{~mL} / \mathrm{min}\right)$. Samples were collected in sampling bottles and stored in a refrigator until analysis. After the extraction, the solid material was removed from the extractor and dried on air.

\subsubsection{Liquid-Liquid Extraction (LLE)}

A hexane-methanol-water system was used in this series of measurements. The experimental plan can be seen in Fig. 2 [33]. This measurement aims to evaluate the effect of the composition on the $\mathrm{CBD}$ distribution ratio of the winterized natural hemp extracts. The five initial molar compositions can be seen in Table 1.

The basis of measurements was the combined dry extracts of all 20-minute PLE experiments. After dissolving the dry extract in ethanol, the liquid was cooled to $273 \mathrm{~K}$ for an hour, then the precipitated waxes of the cold liquid were removed by filtration (winterization). After that, the bulk solution was divided into five equal parts and then stripped dry at $318 \mathrm{~K}$ by the Biotage ${ }^{\mathrm{TM}}$ TurboVap LV. The separated dry materials were weighted. The five dry material samples were dissolved in the five initial liquid-liquid mixture. Ultrasound was employed to enhance the dissolution of the heavier components.

In each step, the samples were mixed thoroughly for 10 minutes then submerged in a water bath set at $298 \mathrm{~K}$ for $1 \mathrm{~h}$. After reaching equilibrium and phase settling a minimal (approximately) $0.5-1 \mathrm{~mL}$ sample was taken

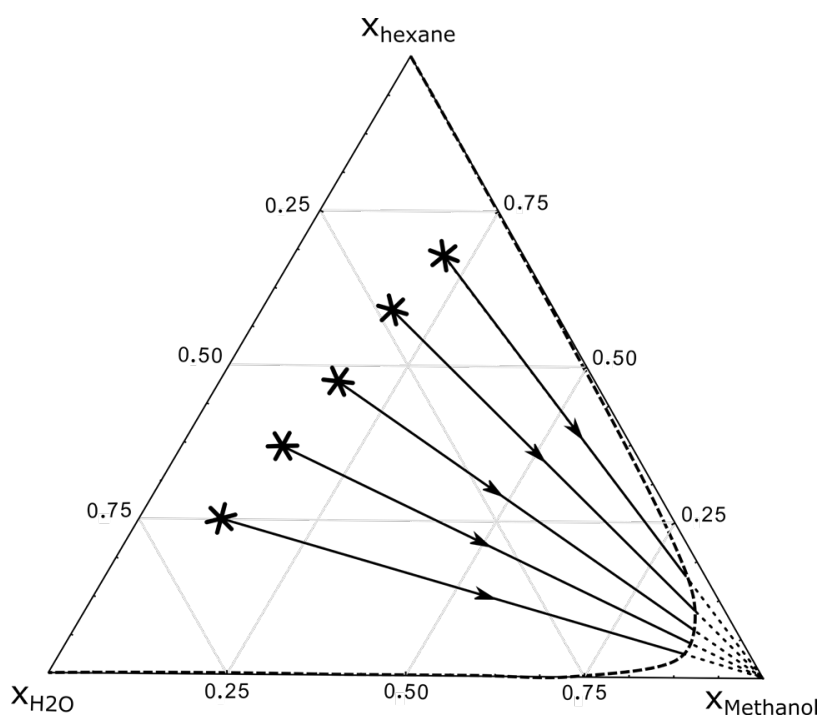

Fig. 2 LLE experimental schematic; *: initial composition, $\rightarrow$ : represents the direction of methanol increase in each run; dashes curve: phase boundary at $298.15 \mathrm{~K}$ reported: [33]

Table 1 Initial molar composition of the liquid-liquid extraction runs

\begin{tabular}{lccc}
\hline & $m_{\text {initial liquid }}=250 \mathrm{~g} ; m_{\text {dissolved dry mat. }}=0.606 \pm 0.045 \mathrm{~g}$ \\
Code & $x_{\mathrm{MeOH}}$ & $x_{\text {Hexane }}$ & $x_{\mathrm{H}_{2} \mathrm{O}}$ \\
\hline 1 & 0.1145 & 0.2511 & 0.6344 \\
2 & 0.1402 & 0.3688 & 0.4910 \\
3 & 0.1646 & 0.4741 & 0.3613 \\
4 & 0.1815 & 0.5904 & 0.2281 \\
5 & 0.2087 & 0.6788 & 0.1125 \\
\hline
\end{tabular}

from each phase. It was weighed, then stored in a refrigerator until analysis. Each new measurement point was achieved by adding to each system a known amount of methanol repeatedly.

At the last measurement point with the highest methanol content, a temperature dependence study was conducted. The samples were additionally kept at $323 \mathrm{~K}$ (water bath) and $278 \mathrm{~K}$ (refrigerator) for 2 and $12 \mathrm{~h}$, respectively, and sampled in the same manner at both temperatures.

\subsubsection{High-Performance Liquid Chromatography (HPLC) method: CBD analysis}

After stripping the samples of the PLE experiments, the weighted dry material was dissolved in methanol until completely dissolved. An ultrasonic bath was used for approx. 10 minutes to guarantee the total dissolution and homogenization. A probe of volume around of $1-2 \mathrm{~mL}$ from the solution was filtered with a $0.02 \mu \mathrm{m}$ syringe filter into a clean vial, and an amount of $100 \mu \mathrm{L}$ of the filtrate was introduced to a clean vial and further diluted with $1.5 \mathrm{~mL}$ of methanol. 
In the case of the LLE experiments, approximately $1 \mathrm{~mL}$ sample was taken from both phases, then weighted. The hexane-rich phase was vacuum distilled at $313 \mathrm{~K}$, to $125 \mathrm{~Pa}$. The water-rich phase was diluted with methanol to 20-times volume, then evaporated with the BiotageTM TurboVap LV. The dry material of both phases was redissolved with $300 \mu \mathrm{L}$ methanol and stored in a vial in a freezer (at $255 \mathrm{~K}$ ) until analysis. The concentration of these samples varied in a wide range. Therefore, initially, $10 \mu \mathrm{L}$ was analyzed and after the results, the dilution ratio -by evaporation and redissolution- was decreased if the concentration was out of calibration range.

Jasco LG-980-02 Ternary Gradient Unit was providing gradient mixing. Jasco PU-1580 Intelligent HPLC pump was used to provide a constant flow rate with an upper-pressure limit of $25 \mathrm{MPa}$ connected to a Jasco AS-2057 plus Intelligent Sampler. $10 \mu \mathrm{L}$ samples were injected (aside from some LLE samples). The components were separated on a $3.9 \times 150 \mathrm{~mm}$ Waters Symmetry ${ }^{\circledR} \mathrm{C} 18$ column with $5 \mu \mathrm{m}$ particle size, kept at $303 \mathrm{~K}$. For compound detection a Jasco MD-910 (Multiwavelength Detector) photo diode array detector was used. For mobile phase a solvent mixture was used in gradient elution mode, the gradient profile is presented below, in the Table 2 .

For quantitation, the CBD concentration had been calibrated with the dilution series of Cerilliant ${ }^{\circledR} 1 \mathrm{mg} / \mathrm{mL}$ CBD standard (product ID: C-045-1ML). The retention time of CBD was 6.5 minutes. Two calibration intervals had been determined. The detection was performed at the wavelengths of $\lambda=215 \mathrm{~nm} 0.0123-0.111 \mathrm{mg} / \mathrm{mL}$ and at $\lambda=230 \mathrm{~nm}, 0.111-1 \mathrm{mg} / \mathrm{mL}$ ranges. The coefficient of determination $\left(R^{2}\right)$ of the calibration lines were 0.9959 and 0.9997 from 3 and 4 measurement points, respectively.

\subsection{Data analysis}

In the PLE experiments the calculation of results were the following:

$$
\begin{aligned}
Y_{n} & =\left(\sum_{i=1}^{n} m_{i}\right) / m_{\mathrm{dry} \text { mat. }} \\
f_{n} & =\left(\sum_{i=1}^{n} m_{i, \text { solvent }}\right) / m_{\text {dry mat. }}
\end{aligned}
$$

wherein Eq. (1) $Y_{n}$ is the yield $[\mathrm{w} / \mathrm{w} \%]$ at measurement point $n ; m_{i}$ is the mass of collected dry sample at measurement point $i[\mathrm{~g}], m_{\text {dry mat. }}$ is the mass of the dry material filled in the extractor column initially [g], and in Eq. (2)
Table 2 Gradient profile of the HPLC method

\begin{tabular}{lcc}
\hline \multicolumn{2}{c}{$\dot{V}_{\text {total }}=1 \frac{\mathrm{mL}}{\min }$} \\
Time [min] & {$[\%]^{\mathrm{a}}$} & {$[\%]^{\mathrm{b}}$} \\
\hline 0 & 0 & 100 \\
1 & 0 & 100 \\
4 & 80 & 20 \\
7 & 80 & 20 \\
9 & 20 & 80 \\
\hline 1 & 0 & 100 \\
\hline
\end{tabular}

a $1: 3 \mathrm{~V} / \mathrm{V}$ mixture of a $25 \mathrm{mM}$ ammoniumacetate aqueous solution with pH 4.7 (set with aceticacid) and Emplura ${ }^{\circledR}$ methanol, respectively;

${ }^{\mathrm{b}}$ Emplura ${ }^{\circledR}$ methanol

$f$ is the feed ratio [-] serving as a time-like variable; $m_{i, \text { solvent }}$ is the used amount of solvent between measurement point $i$ and $i-1[\mathrm{~g}]$.

The modified Brunner equation [34] is applied for the evaluation of the yield curves, defined as:

$$
Y=x_{o} \times\left(1-e^{-k \times f}\right) \text {, }
$$

where $Y$ is the extraction yield [\%]; $x_{o}$ is the total retrievable content of the target component(s) in the same dimension as yield; $k$ is mass transfer-like coefficient [-], $f$ is the feed ratio.

The regression of the Brunner equation was carried out by the optimization of both parameters $x_{o}, k$ for each dataset. The least-squares method was applied to minimize the error, defined as:

$$
\operatorname{Min}\left(\text { Error }^{2}\right)=\sum\left(Y_{f_{n}}^{m}-Y_{f_{n}}^{\prime B r}\right)^{2},
$$

where $Y_{f_{n}}^{m}[\%]$ is the measured yield at feed ratio $n ; Y_{f_{n}}^{B r}$ is the fitted Brunner equation at a feed ratio $n$.

The regressed $x_{o}, k$ values were further studied with Statistica 13 (TIBCO Software. Inc., Palo Alto, CA) to evaluate the relation between the parameters and the independent variables of $(\mathrm{P}, \mathrm{T})$.

In the LLE experiments, the distribution ratio is defined as

$$
K_{D}=\frac{C(\text { organic })}{C(\text { aqueous })},
$$

where $K_{D}[-]$ is the distribution ratio; $C$ (organic) is the concentration of the studied component in the organic phase; $C$ (aqueous) is the concentration of the same component in the aqueous phase. The concentration was given in mass concentration. Molar concentration cannot be used due to the complexity of the plant extracts. 


\section{Results and discussion}

\subsection{Intensified PLE of hemp extraction}

Two sets of total sampling time, 20 and 5 minutes, with 5 and 1 minute continuouse sampling was carried out according to a randomized three-level factorial design with pressure and temperature variables at 8-10-12 MPa and $323-333-343 \mathrm{~K}$ to investigate the effect of the parameters on the total- and CBD yields and to study the technical kinetics of the mass transport in a semi-continuous operation. Three central-point repetitions were included to further validate the results.

There are several mathematical descriptions of extraction processes based on different pore types and numerous assumptions [32-37]. The complexity, mainly the number of fitting parameters and coefficients, varies greatly in different models. One of the common mathematical descriptions of the semi-continuous solid-liquid extraction is based on the modified Brunner equation, see Eq. (3). The two parameters give information about both the total yield and the overall mass transfer.

In this study regression of the modified Brunner equation was carried out by the optimization of both parameters $x_{o}, k$ for the results. The obtained values were further correlated with the independent variables, $P$ and $T$.

\subsubsection{Yield curves and regression analysis}

Considering the literature of batch PLE of hemp threshing residue experiments (extraction times [32]: 10-100 min; [31]: 45 min) 20 minute total sampling with 5 minute continuous sample collection was deemed fit to record the early stages of the extraction. The average errors of fitting by Brunner equation were $0.22 \%$ and $0.01 \%$ for the total- and CBD yield curves, respectively. However, the curves showed that in all cases at least $70 \%$ of the yield was extracted in the first 5 minute period, see Fig. 3. Therefore, both totaland sampling time was further decreased to study the mass transport more accurately.

In the case of 1 minute continuous sampling up to $5 \mathrm{~min}$ utes, the average errors of fitting by Brunner Eq. were $0.60 \%$ and $0.18 \%$ for total- and CBD yields, respectively.

An example of the yield curves can be seen on Fig. 3. Both, total and CBD extraction yield curves are appropriately described with Eq. (3). Despite the good fitting results, both multiple- and response surface regression yielded no correlation with the independent variables when correlated with the fitted $x_{o}, k$ values in Eq. (3) of all experiments (with a probability $<0.05$ ). Most likely the selected pressure and temperature ranges were too narrow to determine any tendencies.

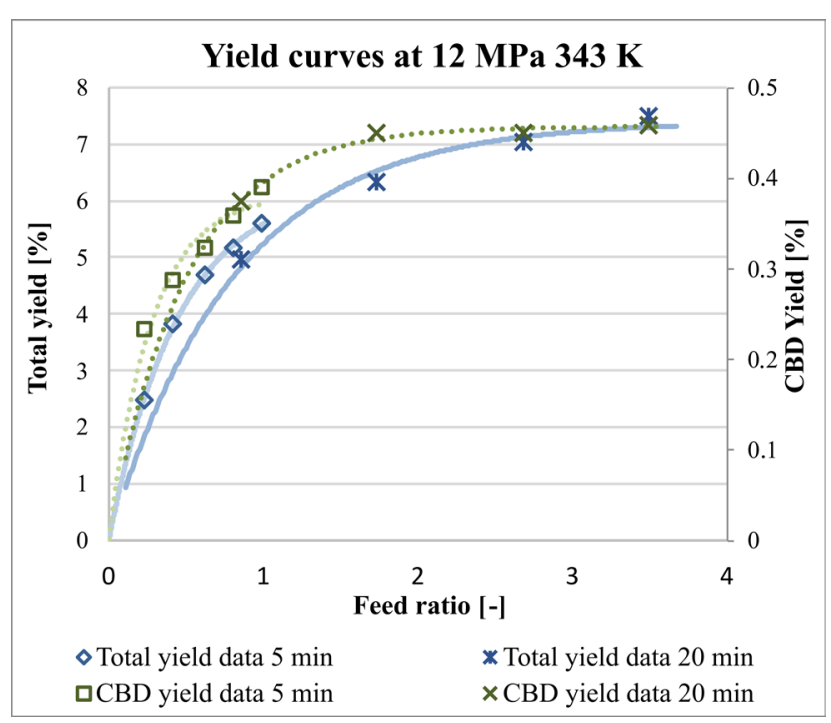

Fig. 3 Experimental data and fitted curves of PLE experiment; continuous lines: total yield fitted by Eq. (3), dotted line: CBD yield fitted by Eq. (3)

The highest CBD content can be achieved at $12 \mathrm{MPa}$, $343 \mathrm{~K}$, and 2.7 feed ratio (approx. $15 \mathrm{~min}$ extraction), seen on Fig. 3. Comparing the best result with the one obtained from a previous study conducted by our SFE research group using the same plant material and carried out $\mathrm{scCO}_{2}$ extraction with $10 \mathrm{w} / \mathrm{w} \%$ of ethanol co-solvent ( $\left.\mathrm{scCO}_{2} \mathrm{EtOH}\right)$ [25], we found the following: in case of PLE with ethanol, the operation time and solvent mass consumption have been decreased 20 and 31-fold, respectively. However, by fast and efficient PLE extraction the maximum CBD yield was $5.1 \mathrm{~g} \mathrm{CBD/g}$ dry material, significantly lower than in the case of the co-solvent assisted supercritical fluid extraction (6.98 g CBD/g dry material). The CBD concentration of the PLE extract was also lower, $6.3 \mathrm{w} / \mathrm{w} \%$ compared to $10.5 \mathrm{wt} \%$ obtained by $\mathrm{scCO}_{2} \mathrm{EtOH}$.

The yield difference is most likely the result of the vast difference in solvent consumption, but it can be also explained by the easier penetration of the supercritical carbon dioxide into the solid matrix. The purity difference can be explained with the polarity difference of the two solvents. The lower CBD concentration of the PLE extracts is in agreement with the expectations. Ethanol dissolves various components from the plant material, while a carbon dioxide-based solvent mixture has a better selectivity towards the semi-polar CBD.

This novel semi-continuous PLE extraction results complete extraction with enhanced productivity with shorter extraction time and lower liquid-to-solid ratio (3 for semi-continuous PLE, 3.3-5 for the batch PLE [32]). 


\subsubsection{Concentration profile of PLE}

If the concentration of the solution obtained during the extraction was plotted against the feed ratio (or time) an exponential correlation was observed. In reality, the concentration of the liquid leaving the extraction column was not known because of the sampling method chosen. The measured values were an average concentration in a time interval. Therefore, plotting the logarithm of the concentration to the arithmetic average of the feed ratio was chosen. The results can be seen in Fig. 4 [25].

The plot shows that both the total extract and CBD concentrations decline rapidly in the early stage (up to feed ratio $\approx 1-1.5$ ). The components on the surface of the plant material were rapidly dissolved and transported to the bulk. Then after the transition phase, the solid-diffusion control started. It was more distinct for the extract concentration profile.

Interestingly, if the results of the $\mathrm{scCO}_{2}-\mathrm{EtOH}$ [25] were compared, at first, no similarity could be observed. However, when the consumed $\mathrm{CO}_{2}$ was excluded from the
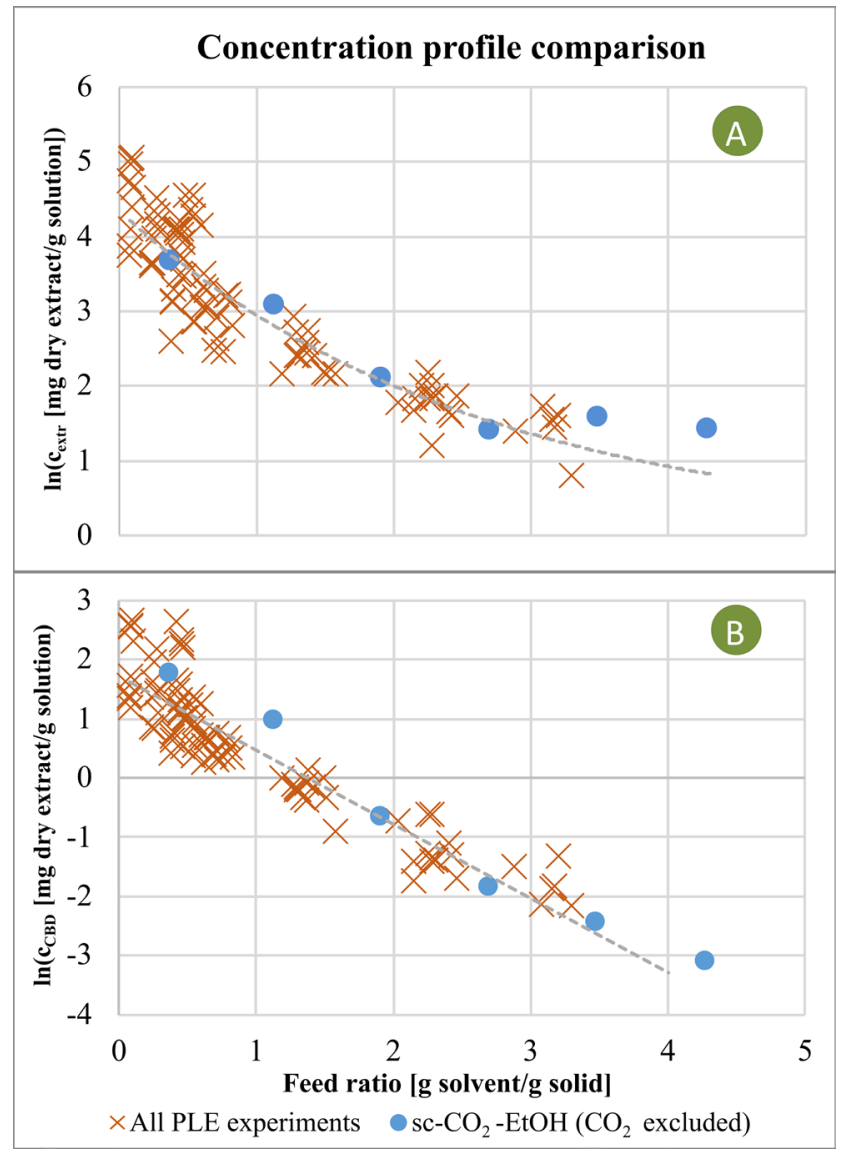

Fig. 4 Logarithmic concentration profiles of all PLE experiments and $\mathrm{scCO}_{2}-\mathrm{EtOH}[25]$ - with exclusion of $\mathrm{CO}_{2}$ from the feed ratio; (a) extract concentration profile, (b) CBD concentration profile; grey dashed lines just guide the eye feed ratio calculations and only the $10 \mathrm{w} / \mathrm{w} \%$ EtOH was considered as a solvent, a unique phenomenon was observed as it can be seen on Fig. 4 (blue dots). Both total extract- and CBD concentration curves of this so-called pseudo-concentration profiles of the semi-continuous supercritical extraction match the PLE profiles. Probably, the mass-transfer from solid to the liquid of the soluble components is highly enhanced by the ethanol. This phenomenon could also explain the more than 30 -fold intensification of solvent mass consumption since the main factor was the ethanol.

\subsection{Results of LLE: distribution ratio results}

After the plant material extraction, the purity of the extracts was typically not suitable for direct application in the cosmetic, pharmaceutical, or pharma industries. Bioactive components are typically heat sensitive, thus Liquid-Liquid Extraction is a common refining method. The distribution ratio (defined by Eq. (5)) is usually up to the experimental determination in all newly charted systems.

Five different compositions of hexane-methanol-water liquid-liquid equilibrium were created to study the distribution ratio of the winterized combined extracts from the PLE experiments with a 20 minute total sampling time. A fixed hexane-water ratio was used in each run and only the methanol content was further increased four times, without entering the single-phase region. A minimal quantity of samples $(0.5-1 \mathrm{~mL})$ were taken from the bulk after equilibration.

Before evaluation, the concentration profile was verified. Constant CBD concentration was not observed in any phase that means, that the solubility limit was not reached. The determined distribution coefficients are presented in Fig. 5.

High methanol dependency of the distribution coefficient can be observed. Increasing the methanol content facilitates the migration of CBD from the hexane-rich phase into the water-rich phase. This is most likely caused by the dual polar-apolar property of methanol thereby promoting the solution of the more apolar CBD in the waterrich phase. Advantageous property of the system is that by simply changing the methanol content, the distribution ratio crosses the $K_{D}=1$ threshold. From an operation perspective, this allows the possibility of a simple back extraction step which can be feasible just by fine-tuning the methanol content between multiple extraction steps.

To further investigate the results a temperature sensitivity study was conducted at the last measurement points (highest methanol content). The results can be seen on Fig. 6 .

On the one hand, small temperature $(\approx 1 \mathrm{~K})$ inhomogeneity throughout the study most likely does not seem to 


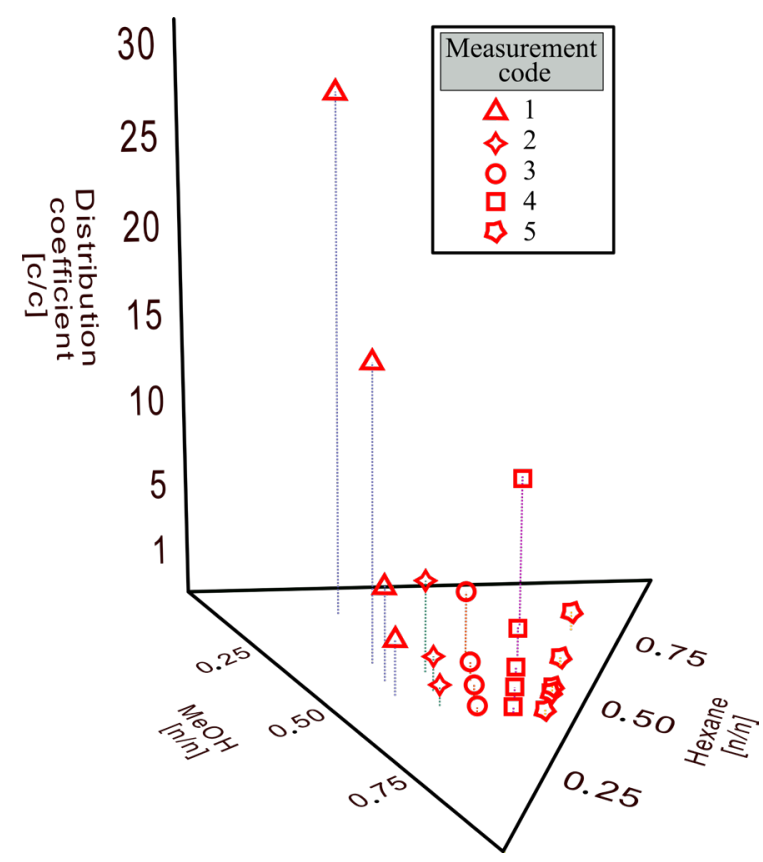

Fig. 5 Distribution coefficient as plotted against the solute-free liquid composition $\left(\mathrm{H}_{2} \mathrm{O}\right.$ axis unmarked for better visibility); for measurement codes: see Table 1

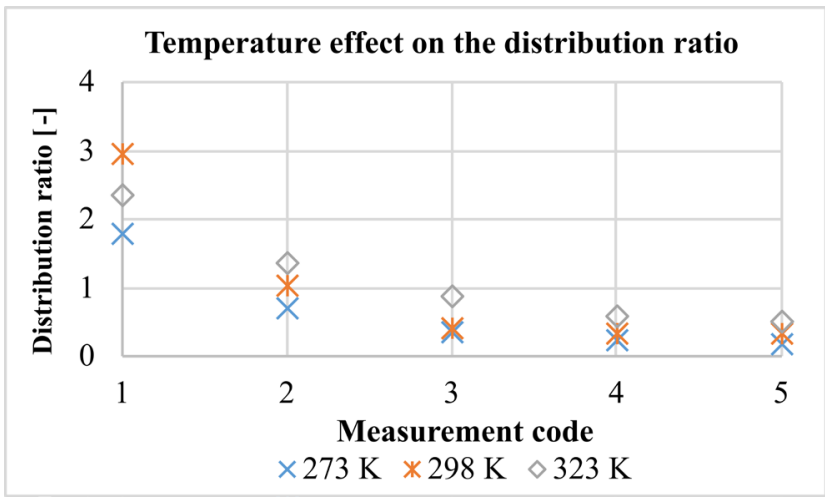

Fig. 6 Temperature dependence study of all samples at the highest methanol content

produce considerable errors in the results, except for experiment with code 1 (see Table 1). In this case, the distribution ratio slightly decreases by the temperature lowering.

\section{References}

[1] Frassinetti, S., Moccia, E., Caltavuturo, L., Gabriele, M., Longo, V., Bellani, L., Giorgi, G., Giorgetti, L. "Nutraceutical potential of hemp (Cannabis sativa L.) seeds and sprouts", Food Chemistry, 262, pp. 56-66, 2018.

https://doi.org/10.1016/j.foodchem.2018.04.078

[2] Li, H.-L. "The origin and use of cannabis in eastern asia linguisticcultural implications", Economic Botany, 28(3), pp. 293-301, 1974. https://doi.org/10.1007/BF02861426
At lower temperature the concentration of CBD in the water-rich phase increases, this could be caused by the low polarity of the CBD. In the case of experiment denoted with code 1 , the result is most likely a measurement error considering the overall tendency.

\section{Conclusion}

In the studied $\mathrm{T}$ and $\mathrm{P}$ intervals the extraction kinetics of semi-continuous pressurized liquid extraction of hemp threshing residue is appropriately described by the modified Brunner equation (see Eq. (3) for both total- and CBD yield curves). The fitting errors does not exceed $1 \%$. The fitting parameters of Eq. (3) were further regressed to the independent variables $P$ and $T$. Neither the extraction yield or rate was influenced by the variables, showing that the semi-continuous PLE with ethanol was a highly robust and efficient extraction method for the hemp treshing residues.

Compared to a previous ethanol and carbon dioxide mixture extraction study of the the same material, intensification was achieved by PLE with pure ethanol solvent. Both, solvent mass consumption and the operation time were decreased more than an order of magnitude in case of PLE. The evaluation of the concentration profiles showed that ethanol is highly facilitating the mass-transfer of all solutes.

The winterized dry extracts were further studied in a methanol-hexane-water system. The results show that the distribution ratio is highly dependent on the methanol content. The ratio equals 1 threshold is crossed which suggests a good back-extraction opportunity after a multistep forward extraction-based purification.

\section{Acknowledgment}

This research was supported by the NRDI Fund (TKP2020 IES, Grant No. BME-IE-BIO) based on the charter of bolster issued by the NRDI Office under the auspices of the Ministry for Innovation and Technology.

[3] Hanuš, L. O., Meyer, S. M., Muñoz, E., Taglialatela-Scafati, O., Appendino, G. "Phytocannabinoids: a unified critical inventory", Natural Product Reports, 33(12), pp. 1357-1392, 2016. https://doi.org/10.1039/c6np00074f

[4] Calzolari, D., Magagnini, G., Lucini, L., Grassi, G., Appendino, G. B., Amaducci, S. "High added-value compounds from Cannabis threshing residues", Industrial Crops and Products, 108, pp. 558-563, 2017.

https://doi.org/10.1016/j.indcrop.2017.06.063 
[5] Tang, K., Struik, P. C., Yin, X., Thouminot, C., Bjelková, M., Stramkale, V., Amaducci, S. "Comparing hemp (Cannabis sativa L.) cultivars for dual-purpose production under contrasting environments", Industrial Crops and Products, 87, pp. 33-44, 2016. https://doi.org/10.1016/j.indcrop.2016.04.026

[6] Lichtfoue, E. (ed.) "Sustainable Agriculture Reviews", Springer, Dordrecht, Netherlands, 2012.

https://doi.org/10.1007/978-94-007-5449-2

[7] The Commission of the European Communities "Commission Regulation (EC) No 2860/2000 of 27 December 2000 amending Regulation (EC) No 2316/1999 laying down detailed rules for the application of Council Regulation (EC) No 1251/1999 establishing a support system for producers of certain arable crops, to include flax and hemp grown for fibre, specifying the rules on set-aside areas and amending the base areas for Greece and Portugal", Official Journal of the European Communities, L 332, pp. 63-75, 2000.

[8] Ranalli, P., Venturi, G. "Hemp as a raw material for industrial applications", Euphytica, 140(1-2), pp. 1-6. 2004

https://doi.org/10.1007/s10681-004-4749-8

[9] Attard, T. M., Bainier, C., Reinaud, M., Lanot, A., McQueenMason, S. J., Hunt, A. J. "Utilisation of supercritical fluids for the effective extraction of waxes and Cannabidiol (CBD) from hemp wastes", Industrial Crops and Products, 112, pp. 38-46, 2018. https://doi.org/10.1016/j.indcrop.2017.10.045

[10] Mechoulam, R., Hanuš, L. O., Pertwee, R., Howlett, A. C. "Early phytocannabinoid chemistry to endocannabinoids and beyond", Nature Reviews Neuroscience, 15(11), pp. 757-764, 2014. https://doi.org/10.1038/nrn3811

[11] Patel, A. D. "Cannabinoids in Neurologic Illnesses", Neurologic Clinics, 39(1), pp. 231-241, 2021. https://doi.org/10.1016/j.ncl.2020.09.012

[12] Braithwaite, I., Bhagavan, C., Doppen, M., Kung, S., Oldfield, K., Newton-Howes, G. "Medicinal applications of cannabis/cannabinoids", Current Opinion in Psychology, 38, pp. 1-10, 2021. https://doi.org/10.1016/j.copsyc.2020.06.002

[13] Alves, P., Amaral, C., Teixeira, N., Correia-da-Silva, G. "Cannabis sativa: Much more beyond $\Delta 9$-tetrahydrocannabinol", Pharmacological Research, 157, Article number: 104822, 2020. https://doi.org/10.1016/j.phrs.2020.104822

[14] Finn, K. (ed.) "Cannabis in Medicine: An Evidence-Based Approach", Springer, Cham, Switzerland, 2020. https://doi.org/10.1007/978-3-030-45968-0

[15] Brighenti, V., Pellati, F., Steinbach, M., Maran, D., Benvenuti, S. "Development of a new extraction technique and HPLC method for the analysis of non-psychoactive cannabinoids in fibre-type Cannabis sativa L. (hemp)", Journal of Pharmaceutical and Biomedical Analysis, 143, pp. 228-236, 2017. https://doi.org/10.1016/j.jpba.2017.05.049

[16] Brighenti, V., Protti, M., Anceschi, L., Zanardi, C., Mercolini, L., Pellati, F. "Emerging challenges in the extraction, analysis and bioanalysis of cannabidiol and related compounds", Journal of Pharmaceutical and Biomedical Analysis, 192, Article number: 113633,2021 https://doi.org/10.1016/j.jpba.2020.113633
[17] Nissen, L., Zatta, A., Stefanini, I., Grandi, S., Sgorbati, B., Biavati, B., Monti, A. "Characterization and antimicrobial activity of essential oils of industrial hemp varieties (Cannabis sativa L.)", Fitoterapia, 81(5), pp. 413-419, 2010. https://doi.org/10.1016/j.fitote.2009.11.010

[18] Grijó, D. R., Piva, G. K., Osorio, I. V., Cardozo-Filho, L. "Hemp (Cannabis sativa L.) seed oil extraction with pressurized n-propane and supercritical carbon dioxide", Journal of Supercritical Fluids, 143, pp. 268-274, 2019.

https://doi.org/10.1016/j.supflu.2018.09.004

[19] Subratti, A., Lalgee, L. J., Jalsa, N. K. "Liquified dimethyl ether (DME): A green solvent for the extraction of hemp (Cannabis sativa L.) seed oil", Sustainable Chemistry and Pharmacy, 12, Article number: 100144, 2019. https://doi.org/10.1016/j.scp.2019.100144

[20] Aladić, K., Jarni, K., Barbir, T., Vidović, S., Vladić, J., Bilić, M., Jokić, S. "Supercritical $\mathrm{CO}_{2}$ extraction of hemp (Cannabis sativa L.) seed oil", Industrial Crops and Products, 76, pp. 472-478, 2015. https://doi.org/10.1016/j.indcrop.2015.07.016

[21] Cai, C., Yu, W., Wang, C., Liu, L., Li, F., Tan, Z. "Green extraction of cannabidiol from industrial hemp (Cannabis sativa L.) using deep eutectic solvents coupled with further enrichment and recovery by macroporous resin", Journal of Molecular Liquids, 287, Article number: 110957, 2019. https://doi.org/10.1016/j.molliq.2019.110957

[22] Ribeiro Grijó, D., Vieitez Osorio, I. A., Cardozo-Filho, L. "Supercritical Extraction Strategies Using $\mathrm{CO}_{2}$ and Ethanol to Obtain Cannabinoid Compounds from Cannabis Hybrid Flowers", Journal of $\mathrm{CO}_{2}$ Utilization, 30, pp. 241-248, 2019. https://doi.org/10.1016/j.jcou.2018.12.014

[23] Valizadehderakhshan, M., Shahbazi, A., Kazem-Rostami, M., Todd, M. S., Bhowmik, A., Wang, L. "Extraction of Cannabinoids from Cannabis sativa L. (Hemp)-Review", Agriculture, 11(5), Article number: 384, 2021.

https://doi.org/10.3390/agriculture11050384

[24] Da Porto, C., Decorti, D., Natolino, A. "Separation of aroma compounds from industrial hemp inflorescences (Cannabis sativa L.) by supercritical $\mathrm{CO}_{2}$ extraction and on-line fractionation", Industrial Crops and Products, 58, pp. 99-103, 2014. https://doi.org/10.1016/j.indcrop.2014.03.042

[25] Vági, E., Balázs, M., Komoczi, A., Mihalovits, M., Székely, E. "Fractionation of phytocannabinoids from industrial hemp residues with high-pressure technologies", The Journal of Supercritical Fluids, 164, Article number: 104898, 2020. https://doi.org/10.1016/j.supflu.2020.104898

[26] Benelli, G., Pavela, R., Lupidi, G., Nabissi, M., Petrelli, R., Ngahang Kamte, S. L., ..., Maggi, F. "The crop-residue of fiber hemp cv. Futura 75: from a waste product to a source of botanical insecticides", Environmental Science and Pollution Research, 25(11), pp. 10515-10525, 2018. https://doi.org/10.1007/s11356-017-0635-5 
[27] Vági, E., Balázs, M., Komóczi, A., Kiss, I., Mihalovits, M., Székely, E. "Cannabinoids Enriched Extracts from Industrial Hemp Residues", Periodica Polytechnica Chemical Engineering, 63(2), pp. 357-363, 2019. https://doi.org/10.3311/PPch.12896

[28] Bertoli, A., Tozzi, S., Pistelli, L., Angelini, L. G. "Fibre hemp inflorescences: From crop-residues to essential oil production", Industrial Crops and Products, 32(3), pp. 329-337, 2010. https://doi.org/10.1016/j.indcrop.2010.05.012

[29] Pavlovic, R., Panseri, S., Giupponi, L., Leoni, V., Citti, C., Cattaneo, C., Cavaletto, M., Giorgi, A. "Phytochemical and Ecological Analysis of Two Varieties of Hemp (Cannabis sativa L.) Grown in a Mountain Environment of Italian Alps", Frontiers in Plant Science, 10, Article number: 1265, 2019. https://doi.org/10.3389/fpls.2019.01265

[30] Fathordoobady, F., Singh, A., Kitts, D. D., Pratap Singh, A. "Hemp (Cannabis sativa L.) Extract: Anti-Microbial Properties, Methods of Extraction, and Potential Oral Delivery", Food Reviews International, 35(7), pp. 664-684, 2019. https://doi.org/10.1080/87559129.2019.1600539

[31] Kitrytė, V., Bagdonaitè, D., Rimantas Venskutonis, P. "Biorefining of industrial hemp (Cannabis sativa L.) threshing residues into cannabinoid and antioxidant fractions by supercritical carbon dioxide, pressurized liquid and enzyme-assisted extractions", Food Chemistry, 267, pp. 420-429, 2018. https://doi.org/10.1016/j.foodchem.2017.09.080
[32] Serna-Loaiza, S., Adamcyk, J., Beisl, S., Kornpointner, C., Halbwirth, H., Friedl, A. "Pressurized Liquid Extraction of Cannabinoids from Hemp Processing Residues: Evaluation of the Influencing Variables", Processes, 8(11), Article number: 1334, 2020. https://doi.org/10.3390/pr8111334

[33] Liu, J., Qin, Z., Wang, J. "Liquid-Liquid Equilibria for Methanol + Water + Hexane Ternary Mixtures", Journal of Chemical \& Engineering Data, 47(5), pp. 1243-1245, 2002. https://doi.org/10.1021/je0255269

[34] Brunner, G. "Mass Transfer from Solid Material in Gas Extraction", Berichte der Bunsengesellschaft für physikalische Chemie, 88(9), pp. 887-891, 1984. https://doi.org/10.1002/bbpc.19840880923

[35] Sovová, H. "Rate of the vegetable oil extraction with supercritical $\mathrm{CO}_{2}-\mathrm{I}$. Modelling of extraction curves", Chemical Engineering Science, 49(3), pp. 409-414, 1994. https://doi.org/10.1016/0009-2509(94)87012-8

[36] Peker, H., Srinivasan, M. P., Smith, J. M., McCoy, B. J. "Caffeine extraction rates from coffee beans with supercritical carbon dioxide", AIChE Journal American Institute of Chemical Engineers, 38(5), pp. 761-770, 1992. https://doi.org/10.1002/aic.690380513

[37] Crank, J. "The Mathematics of Diffusion", Oxford University Press, London, UK, 1979. 